\title{
Entretien avec Yvonne Mété-Nguemeu, auteur de Femmes de Centrafrique, âmes vaillantes au cour brisé
}

Propos recueillis par Ngozi Obiajulum Iloh, Université du Benin, Nigéria

NOI : Pouvez-vous nous donner un peu de votre biographie? La date de votre naissance, votre scolarisation, etc.

YM-N : Je suis née le 10 décembre 1956 à Sibut, une ville située à $185 \mathrm{~km}$ de Bangui, la capitale de la République Centrafricaine. J'y ai passé mon enfance et fréquenté l'école primaire catholique jusqu'au CM2 (Cours Moyen élémentaire). Ensuite j'ai fréquenté le collège et le lycée Pie XII à Bangui. Après avoir obtenu le baccalauréat B en 1979, j'ai obtenu une bourse d'étude pour venir poursuivre mes études supérieures en France où je vis depuis lors avec des séjours réguliers dans mon pays d'origine.

NOI : A travers votre livre, que je considère comme une autobiographie, vous avez parlé des femmes de Centrafrique, est-ce que vous le considérez comme un roman authentique ? Je pose cette question parce qu'au début, vous avez commencé par votre naissance et votre enfance, plus tard, on constate un décalage au moment où vous êtes partie pour la France.

YM-N : Mon livre raconte l'évolution de la société centrafricaine telle que je l'ai vécue et constatée tout au long de ma vie. Toutes les histoires et faits relatés sont réels. Cependant j’ai modifié certains faits pour éviter que des personnes puissent être reconnues et pointées du doigt (ex : les femmes violées qui essaient de se reconstruire ...). Je précise comme je l'ai fait dans le livre que les «Femmes de Centrafrique » dont il est question dans le livre sont : des centrafricaines de naissance, d'adoption, de cœur. Elles sont soient des expatriées arrivées en Centrafrique par vocation pour enseigner aux enfants centrafricains, ou des épouses de centrafricains ; ou encore nées en Centrafrique. Le livre ne parle que de mes expériences en République Centrafricaine, d'où ce décalage dans la chronologie.

NOI : Je constate que c'est difficile de vous séparer de votre association Centrafrique Sans Frontières car votre livre est aussi basé sur les expériences vécues ainsi que des expériences des femmes de chez vous. Pouvez-vous séparer les deux ?

YM-N : J'aurai du mal à séparer les deux pour le moment, car l'association fait partie de ma vie. Ce livre est une façon de faire le bilan de la première phase du projet associatif. Il m'aide à réfléchir sur la meilleure façon de soutenir ces femmes qui n'ont pas eu la possibilité de 
choisir la vie qu'elles auraient souhaitée. Tant que je n'aurai pas réalisé une infime partie de ce que j'espère faire pour ces femmes, l'association sera toujours là pour me rappeler mes engagements.

NOI : Le Centrafrique est un pays méconnu du point de vue international, voire en Afrique. Pourtant, vous avez choisi comme titre «Femmes de Centrafrique... » afin de faire connaitre votre pays au monde international. Je crois que cela a vraiment marché. Dites-nous alors ce qui vous a poussé d'ajouter le nom de votre pays.

YM-N : Justement, comme vous l'avez si bien dit, mon pays n'existe pas tellement sur le plan international. Dans les années quatre-vingts, juste après la chute du pouvoir du tristement célèbre dictateur «Bokassa » (octobre 1979), en France quand on te demandait d'où tu étais originaire et que tu répondais «de la République Centrafricaine », on te redemandait «le centre de l'Afrique, mais d'où exactement? »; et si tu précisais « du pays de Bokassa », une bonne partie des personnes te disaient « Ah le pays des diamants?» se rappelant du scandale des diamants qui avait touché un ancien président français.

Mais avec le temps, la nouvelle génération qui n'a pas vécu cette époque, ne sait pas du tout de quel pays il s'agit. Dans ma ville et ma région d'adoption Besançon et la Franche-Comté, j'ai dû mettre ce nom à l'association pour une meilleure visibilité et connaissance géographique. Concernant le livre, c'est un appel lancé en direction des femmes africaines et du reste du monde pour leur dire que nous existons et faisons tout ce que nous pouvons pour être visibles afin de pouvoir suivre leurs pas, même si des fois nous avons l'impression d'avancer à contre courants !

NOI : Votre livre est tellement riche en thèmes couvrant tous les aspects de la condition féminine. Quel est vraiment la place de la femme dans la famille centrafricaine à l'heure actuelle?

YM-N : Imaginez un petit être aux pouvoirs surnaturels qui réussirait à soulever plusieurs objets pesant chacun trois ou quatre fois son poids et arriverait à les maintenir en hauteur pour éviter qu'ils tombent et ne se brisent. C'est exactement ça, la place de la femme centrafricaine : toute la structure familiale tient grâce à l'exploit et au sacrifice de la femme centrafricaine. Son rôle c'est : $80 \%$ des travaux champêtres et $100 \%$ de travaux domestiques ; $100 \%$ de commerce de survie, la maternité et les risques sur sa santé dans un pays où la situation sanitaire est catastrophique, la survie des enfants, du mari, les places mortuaires, elles sont également infirmières (la femme doit trouver des solutions pour guérir mari et 
enfants, car il y a pénurie de médicaments et de médecins et nos hôpitaux ressemblent souvent plus à des mouroirs qu'à des lieues pour sauver des vies humaines ) ...

Mais elle reste persuadée qu'elle ne sert pas à grand-chose et qu'elle ne survit que grâce à l'homme.

NOI : Pourriez-vous nous expliquer votre avis sur les réalités féminines d'aujourd'hui en Centrafrique?

YM-N : C'est un pays dans lequel les droits de femmes ne sont que théoriques. Au maximum $1 \%$ des femmes occupent des postes valorisants. Quand à celles qui accèdent à des postes politique ou de pouvoir, leur influence dans les projets pour améliorer le sort de leurs sœurs est inexistante ou peu visible. La violence faite aux femmes dans la vie de tous les jours laisse indifférent et j'en passe car ce sera tout un livre à écrire. Le sens des manifestations pour les droits des femmes et leur émancipation n'est pas compris par les organisateurs

NOI : Existe-il des différences entre la vie de la femme jadis et la vie de la femme à l'heure actuelle ?

YM-N : Bien sûr, c'est ce que j'ai décrit dans le livre. La femme de jadis a vécu en harmonie avec ce que son époque lui offrait. Elle ne pouvait donc pas connaitre la frustration du fait d'être écartée de quelque chose qu'elle ne connaissait pas. De nos jours, beaucoup de femmes n'ont pas accès à l'école. Ont des grossesses précoces et beaucoup meurent des suites d'accouchement. L'insécurité règne sur presque tout le territoire et le viol est un moyen de pression et de destruction massive d'une population, alors que le viol était condamné sévèrement dans le temps et que les violeurs étaient exclus de la communauté.

NOI : La République Centrafricaine a été ravagée par des guerres et des viols. Parlez un peu de ce phénomène de viol et de la pandémie du sida.

YM-N : Ce phénomène est devenu visible en 2002, au moment où l'ancien président déchu, Monsieur Ange Félix Patasse a fait appel aux miliciens du Mouvement de Libération du Congo (MLC) commandé par le tristement célèbre Jean-Pierre Bemba. Ces rebelles, dont la majorité est contaminée par le sida, a utilisé le viol systématique des femmes et fillettes comme arme de guerre déjà utilisé dans le pays d'origine, la République Démocratique du Congo. Ils ont ciblé toute la population désignée par le président comme lui étant hostile et c'est leur façon de détruire la descendance de cette population. L'impact de nos jours c'est que, dans certaines régions et villes, toute une génération de femmes et fillettes a disparue. 
NOI : Croyez-vous que ce sont les viols qui sont les causes du sida?

YM-N : Le sida avait déjà commencé à se propage avant les viols, par contre ils ont contribué à sa propagation fulgurante par la suite.

NOI : Pourriez-vous nous renseigner mieux sur votre cher pays ?

YM-N : Le voici en résumé: La République Centrafricaine est une ancienne colonie française à partir de 1905 sous le nom de l'Oubangui Chari. Elle a acquis son indépendance en 1958 et est devenue «République Centrafricaine». C'est un pays enclavé situé à l'extrémité orientale du Sahel, entouré par le Soudan à l'est, le Tchad au nord, le Cameroun à l'ouest, et le Congo et la République démocratique du Congo au Sud. Superficie : 622984 km2 ; Population : à peine 4 millions. Ses atouts : fer, coton, bois, diamants, café. Zone de transit du centre de l'Afrique. Bon climat équatorial avec une bonne pluviosité. Ses faiblesses : pas d'accès à la mer, pauvreté des infrastructures routières et absence de réseau ferroviaire. Et des dictatures qui se succèdent avec une mauvaise gouvernance qui s'est installée durablement.

NOI : Dans votre livre, je trouve la narration de la solidarité féminine très intéressante, là où les femmes sont venues au secours de Yaya Marie, cela fait ressortir l'aspect positif de la femme africaine qui n'est plus victime mais prend sa libération dans ses mains ; pourriezvous donner davantage des exemples de telles expérience?

YM-N : Ce serait trop long, mais juste un exemple : beaucoup de veuves du sida sont souvent des rescapées de foyers polygames ayant perdu leur mari et leurs coépouses. J'ai remarqué que la majorité d'entre elles avaient recueilli les enfants de leurs coépouses décédées, quelle que soit la qualité de la relation qu'elles ont eu entre elles du vivant de ces femmes. Face à la dégradation de la situation économique, c'est dans tous les cas, le seul moyen pour ces enfants de ne pas se retrouver à la rue.

NOI : Votre père ne voulait pas que vous soyez mariée dans le livre; pourtant, vous avez trois enfants, est-ce que vous êtes mariée ? Et quel est votre statut maintenant ? Avez-vous donc respecté le désir de votre cher père?

YM-N : Je n'ai jamais été mariée et c'est un choix personnel que j'ai fait selon mon parcours. Le point de vue de mon père ne m'a pas vraiment influencé. Je pense que ce n'est que pure coïncidence si j'ai choisi de vivre seule. En France, mon pays d'adoption, les femmes ont le choix de leur vie et peuvent s'en sortir seules. J'ai eu la chance de vivre dans un pays qui a 
comme devise «la liberté » avec des lois qui la protège, cela aurait été dommage vu mon tempérament de ne pas en profiter. Avec le recul, je me demande si mon père n'était pas visionnaire et n'avait pas compris en m'observant toute petite que je n'étais pas faite pour le mariage. Il n'était pas contre le mariage en général vu qu'il a été un mari fidèle, monogame dans un pays de polygamie, et un bon père.

NOI : Votre livre peut être considéré comme une œuvre anthropologique et sociologique. Vous avez parlé de la religion et mon cher pays, le Nigéria, a été cité comme l'un des pays où le culte chrétien charismatique est répandu. Il y-avait-il une exploitation féminine de la religion? Pourquoi les femmes sont-elles souvent les adhérentes de ces cultes ? Il y-a-il des hommes ?

YM-N : Ces religions ont proliféré en Centrafrique en profitant de la situation économique désastreuse et de la crédibilité d'une population qui n'a plus de repère et qui cherche désespérément à survivre. A partir du moment où la misère règne, que les jeunes n'ont pas de travail, donc un avenir incertain avec un taux de chômage dépassant les $80 \%$, et que les conditions du travail agricole sont difficiles, la voie est libre pour ces prêcheurs. Des individus débarquent et disent représenter une religion qui accomplit des miracles. L'Africain, habitué à se faire racketter sans contrepartie par la police, ses dirigeants et l'administration, tombe dans le panneau car, pour lui, Dieu est le garant et sa situation peut s'améliorer sans effort et de façon pérenne. Les femmes sont souvent les adhérentes de ce culte, car elles comptent sur l'aide divine pour choisir l'homme idéal à leur place, car Dieu seul est infaillible et elles se sont tellement trompées en se fiant à leur instinct ou aux choix de leurs parents.En plus, c'est elles qui sont en majorité ciblées dans les prêches, car elles détiennent en majorité la bourse familiale. Tous les commerces centrafricains sont tenus pour $95 \%$ par les femmes. Les hommes sont minoritaires, car ils ont moins d'argent à donner à ces prédicateurs.

NOI : Le phénomène de clitoridectomie existe il toujours en Centrafrique ? Les raisons poussées par nos mères tiennent-elles toujours en ce moment?

YM-N : L'excision a beaucoup reculé en Centrafrique depuis quelques années, surtout dans les grandes villes. Cependant, elle continue à être pratiquée officieusement, donc en cachette dans les villages, surtout les plus reculés. Elle est si discrète qu'il devient de plus en plus difficile d'identifier les auteurs afin de les punir. Je pense qu'il y a encore du travail à faire et que ce travail passe par l'instruction qui malheureusement est en chute libre. 
NOI : Parlons un peu de l'histoire inconnue de la Centrafrique.

YM-N : Résumé de l'histoire de Centrafrique :_Les premiers habitants de Centrafrique sont les pygmées._La ville de Bangui, capitale actuelle, a été fondée sur la rive du fleuve Oubangui par les Français en 1889. L'Oubangui Chari devient une colonie française. La république centrafricaine a été proclamée le $1^{\mathrm{er}}$ décembre 1958. Le premier président Barthélemy Boganda a été tué dans un accident d'avion le 29 mars 1959. Son indépendance a été proclamée le 13 aout 1960. David Dacko, cousin de Boganda devient alors président. Il sera renversé par Jean-Bedel Bokassa qui s'empara du pouvoir grâce à un coup d'Etat en 1965._Le 4 décembre 1976 Bokassa se fit couronner Empereur Bokassa $1^{\mathrm{er}}$ de Centrafrique et vida les caisses de l'Etat en organisant une cérémonie grandiose. Il mit en place une dictature très répressive et organisa le massacre d'élèves et étudiants qui s'étaient soulevés contre sa politique en avril 1979._La France organisa alors l'opération Barracuda et le renversa en remettant l'ex-président David Dacko en septembre 1979. Bref, André Kolingba le renverse deux ans plus tard et organise des élections sous contrainte en 1993; Ange Félix Patasse prend le pouvoir jusqu'en 2003 où il sera renversé à son tour par le président actuel François Bozize. Et voilà, rien de bien réjouissant à part que la mauvaise gouvernance s'est installée et que le pays connait une descente aux enfers à chaque changement de régime.

NOI : Pourquoi l'Afrique méprise-t-elle ses vieillardes ? Est-ce causé par la pauvreté ?

YM-N : On méprise de plus en plus les vieillards en Afrique avec l'évolution de la société qui s'ouvre sur le monde et dont le fonctionnement a changé. Dans les villes, les gens imitent l'Europe et réduisent la notion de la famille à celle de l'occident : le couple et ses enfants. Or, l'Occident y est arrivé progressivement après avoir créé des structures d'accueil aux personnes âgées et à leur mode de fonctionnement, ce qui n'existe pas chez nous en Afrique. Le ravage du sida a accéléré le phénomène avec des générations disparues. Les adultes de cinquante ans et plus, qui devraient prendre soin de leurs parents âgés, ont été des victimes du sida. Beaucoup de jeunes orphelins du sida ont grandi sans parents et grands-parents et n'ont pas cette éducation du respect des personnes âgées. En plus de ça, les familles ont de moins en moins la capacité de nourrir beaucoup de bouches et se referment sur elles. Notre société s'éloigne fatalement du traditionnel respect des personnes âgées, de sa générosité et de son sens de l'accueil généreux que l’Occident lui envie tant. 
NOI : Maintenant que vous êtes auteure d'un livre, attend-on une autre œuvre de vous?

YM-N : J'aurais déjà sorti mon deuxième livre si je n'avais pas eu des soucis de santé depuis deux ans. J'ai beaucoup de projets de livres et quelques écrits sur mon ordinateur, mais le temps jusque-là me manque. Mon projet associatif me prend beaucoup de temps, car la situation des femmes s'est beaucoup dégradée et j'aimerais trouver une solution à proposer, surtout aux femmes des milieux ruraux pour qu'elles puissent s'en sortir avec les produits locaux. Je vis encore en France et je dois continuer à travailler pour ma retraite, le financement des projets et le loyer ; tout cela me laisse peu de temps pour écrire.

NOI : Je crois qu'avec votre ONG vous pouvez publier d'autres histoires des femmes de chez vous.

YM-N : J'aime beaucoup écrire sur les femmes africaines et surtout centrafricaines, car leur histoire est méconnue et je préfère parler de ce que je maitrise vraiment plutôt que de faire des suppositions. C'est pourquoi j'écrirai plus sur la réalité des femmes pour leur rendre hommage, car j'admire la vie de ces femmes, leur courage, leur sens de responsabilité et leur engagement.

\section{Deuxième tranche}

NOI : Pouvez-vous continuer le bilan de l'évolution des conditions de la vie des femmes depuis l'époque de votre mère jusqu'à nos jours?

YM-N : Le bilan de l'évolution des conditions de la vie des femmes en République Centrafricaine est très mitigé. Les femmes n'ont pas réussi à profiter de l'évolution de la société pour se tailler une part de roi aux postes clés. Ce tremplin qui aurait pu non seulement leur donner le pouvoir d'améliorer durablement leur avenir, mais aussi celui de leurs semblables et transformer positivement la société centrafricaine.

NOI : Il y a-t-il vraiment eu évolution?

YM-N : Je ne parlerai pas d'évolution, juste que certaines femmes ont pu avoir la possibilité de changer les choses, mais elles n'ont pas su utiliser cette chance. Nous avons manqué «notre rendez-vous » avec l'histoire.

NOI : Vous pouvez en passer à l'époque coloniale ?

YM-N : Les femmes centrafricaines sont restées coincées dans cette époque coloniale et le fait que certaines ont eu la chance d'avoir une instruction, n'a pas changé grand-chose à leur 
destin. Nous demeurons sur la touche, car nous avons choisi de nous contenter des miettes de table de ceux qui font la pluie et le beau temps, c'est-à-dire des politiciens adeptes de la mauvaise gouvernance.

NOI : Quel est le destin des femmes centrafricaines?

YM-N : Le destin des femmes centrafricaines est de bâtir un pays représentatif des richesses qui abondent dans ses sous-sols et ses forêts, afin de rendre son peuple libre et le faire entrer dans l'histoire. Pas de rester à la traine de l'humanité. Un proverbe africain dit ceci « Si tu ne peux: Protéger le peuple et braver l'ennemi, / Donne ton sabre de guerre aux femmes, /Qui t'indiqueront le chemin de l'honneur... Je pense que c'est aux femmes de comprendre quand le moment est venu pour elles de prendre ce sabre et d'agir pour le peuple. Elles doivent devenir actrices et non attendre qu'on leur fasse l'aumône. A elles de devenir des « indignées » comme l'a pensé un grand homme français.

NOI : Est-ce que le destin des femmes reste seulement à leur autonomisation ? Laquelle spécifiquement?

YM-N : Le destin des hommes est le même que celui des femmes; je ne pense pas qu'il y ait deux destins distincts. Sauf que les premiers cherchent toujours à dominer les autres et à privilégier leur ego, alors que les deuxièmes passent leur vie à interroger leur conscience sur les conséquences de leurs actions fictives, et finissent par renoncer à agir par peur de l'échec ! La majorité des femmes sont matériellement autonomes, c'est leur esprit qui reste dépendant. Elles ont du mal à assumer cette autonomie, d'où leur faiblesse.

NOI : Je crois qu'il y un lien entre la pauvreté, l'analphabétisme et l'autonomisation. Etesvous d'accord?

YM-N : Je vois plus un lien entre la pauvreté, l'inaction et le manque d'esprit d'indépendance. Nos mères étaient analphabètes mais arrivaient à mener une vie convenable comparée au niveau de vie de leur temps. De nos jours, beaucoup de femmes lettrées vivent dans la misère totale, car elles ont baissé les bras. Nous vivons en permanence dans une sorte de prison intellectuelle et c'est ce qui entrave notre capacité d'action.

NOI : Croyez-vous à l'émancipation de la femme ?

YM-N : Bien sûr, l'émancipation de la femme est une arme très sensible. L'essentiel réside dans cette maîtrise et l'utilisation que la femme en fait, ainsi que son ambition personnelle. 
NOI : N’y-a-il pas de femmes émancipées en Centrafrique?

YM-N : Il y a des femmes émancipées qui ont peur de s'assumer pleinement sans l'accord du mâle. Elles ont peur d'être rejetées ou traitées de féministes, voire de mauvaises femmes. L'émancipation de la femme centrafricaine a toujours été sous-utilisée et n'a donc pas pu être efficace.

NOI : Êtes-vous féministe ? Vous êtes de quelle école idéologique, womaniste, motheriste, stiwaniste, etc. ?

YM-N : Je suis une féministe dont les ailes ont été brulées trop tôt et qui a eu peur de prendre son envol afin d'écrire un pan de l'histoire de son pays. J'ai passé mon temps à prendre mon passé comme prétexte à l'inaction et je le regrette, car je ne saurai jamais si j'aurais pu faire quelque chose de concret pour les autres. Je n'ai aucune disposition à être une femme au foyer; je pense que je serais certainement morte d'ennui. J'ai fait de mon mieux pour transmettre mes rêves à mes enfants, aux jeunes ici ou là. Mais je continue à croire en la force des femmes et je pense que lorsque la femme centrafricaine se réveillera et décidera de faire passer sa passion et son Amour pour son pays avant tout autre considération, le ciel centrafricain s'illuminera.

NOI : Il y a-t-il des différences entre la condition des femmes centrafricaines et celle d'autres pays africains?

YM-N : C'est possible, car la femme centrafricaine n'a pas confiance en elle et a peur d'avoir comme ennemie une autre femme. Pourtant, la solidarité féminine existe, mais elle s'est fixé des limites qui ne lui permettent pas de se surpasser et de donner le meilleur d'ellemême.

NOI : Il y a-t-il une spécificité particulière d'une ou plus des conditions féminines centrafricaines?

YM-N : La condition féminine centrafricaine souffre de manque de volonté de la part des concernées. Je pense que si les femmes centrafricaines le voulaient, elles s'impliqueraient efficacement dans la vie politique et décideraient d'un véritable changement pour l'avenir de leur pays. Celles qui ont été accueillies dans la sphère du pouvoir manquent de volonté et d'ambition politique. Une bonne partie de celles qui ont la capacité de faire bouger les choses, finissent par faire taire la petite voix qui parle en elles. 
NOI : Pourriez-vous donner plus de renseignements sur la prostitution en République centrafricaine?

YM-N : La prostitution est un phénomène qui touche beaucoup la jeunesse, surtout dans les grandes villes et certaines villes commerçantes. Les femmes sont obligées d'assumer les responsabilités domestiques depuis les crises politiques successives et les maris posent de moins en moins des questions sur l'origine de l'argent qui les fait vivre. Il y a eu aussi des manifestations agricoles organisées dans différentes villes qui faisaient se déplacer des fonctionnaires sans leurs femmes. Ceux-ci étaient demandeurs et les jeunes filles de campagne avaient besoin d'argent avec une ignorance totale du fléau qu'est le sida. Certains parents, pour résoudre le problème de pauvreté, sacrifient leurs fillettes en leur confiant de petites denrées à vendre en pleine nuit ou en vendeuses ambulantes, refusant ainsi de voir ce qui se passe. Petit à petit, la prostitution s'est faite une bonne place dans ce pays chrétien aux mille et une églises !!!

NOI : Les guerres civiles, les viols et la pauvreté, n'ont-ils pas contribué à la prostitution ?

YM-N : Les viols ont participé très peu à la prostitution, car à mon avis, les victimes de viol sont tellement détruites qu'elles ont des difficultés à offrir leur corps volontairement, quelles qu'en soient les raisons. La pauvreté qui découle de la mauvaise gouvernance, y est sans doute pour quelque chose.

NOI : Quelle est la place de l'enfant mâle dans la société centrafricaine ? Et votre frère aîné qui est allé poursuivre ses études en France ? Comment se débrouille-t-il ?

YM-N : Le mythe de l'enfant mâle a encore un bel avenir devant lui, même si de plus en plus de parents ont compris que leurs rejetons ne sauveront pas leur vieillesse. C'est comme le paradis : nombreux sont ceux qui y croient, mais quel sera le nombre réel de ceux qui y accèderont. Dieu seul le sait. Mon frère est rentré, a travaillé dans la fonction publique, est devenu ministre et ne l'est plus. Il n'a pas fait mieux que les autres.

NOI : Parlons de la responsabilité économique de la femme centrafricaine ; que font les hommes?

YM-N : Beaucoup d'hommes se sont résignés face à la crise, surtout dans la capitale où beaucoup y étaient venus dans le but de devenir « haut fonctionnaires ». Malheureusement on continue à mettre dans la tête des femmes que vivre seule est une tare ou un péché. Certaines galèrent et passent toute leur vie à élever leur mari et leurs descendants. Cependant dans les 
provinces, on peut constater que beaucoup d'hommes, même polygames participent aux travaux champêtres et le petit commerce est tenu autant par des femmes que par des hommes.

NOI : «Femmes vaillantes », pourquoi avez-vous choisi un tel thème ? Pourtant, il s'agit de la victimisation de ces femmes; n'il y a-t-il pas de contradiction entre la victimisation et la vaillance?

YM-N : Le livre Femmes de Centrafrique... s'adresse à toutes les femmes, qu'elles soient centrafricaines de naissance ou européennes d'origine et centrafricaines d'adoption. C'est le constat d'un déséquilibre entre l'effort consenti par les femmes pour ce pays et le peu de résultat qui en découle. Tout l'Amour que les mères donnent à leurs enfants mâles et le peu de satisfaction et de fierté qu'elles en retirent, est source de déception. C'est plus un rapport entre la mère éducatrice, l'investissement dans une cause pour voir son enfant grandir et qui échoue. C'est plus le cœur brisé de mère, ou d'enseignante, d'éducatrice et moins de relation de couple.

NOI : Vous avez trois filles et aucun garçon. Ne sentez-vous pas de regret de ne pas avoir enfanté de garçon?

YM-N : J'ai désiré des filles les deux premières fois ; ensuite, j'ai voulu une troisième enfant et, cette fois, je m'étais dit que je voulais la surprise. Je pense qu'inconsciemment j'ai toujours eu envie de n'avoir que des filles. Ai-je eu raison ou pas, c'est trop tard pour me poser la question. J'ai fait de mon mieux pour apprécier les joies et surmonter les épreuves que Dieu et la vie m'ont réservées.

NOI : L'intériorisation du complexe d'infériorité chez la femme africaine est un phénomène patriarcal. Qu'en pensez-vous ?

YM-N : La femme africaine admire son père et elle craint de ne pas retrouver ces qualités chez son futur mari ou espère ne pas faire la même pioche (le même choix) que sa mère.

Elle renonce dès le départ à être exigeante dans son choix. Elle se dit : « S'il me faut sauter le pas, à quoi bon me fatiguer à chercher un bon! Ils se valent certainement tous ». Elle ferme les yeux, refuse de rêver et s'engage dans cette aventure.

NOI : Revenons à votre père ; je n'arrive pas à comprendre la composition de votre nom de famille, Mété-Nguemeu ? Expliquez-nous. 
YM-N : Mon père s'appelait Mété et sa mère Nguemeu. Il avait voulu rendre hommage à sa mère en me donnant son nom de Nguemeu comme deuxième nom.

NOI : Qu'est-ce qui vous a inspirée à écrire un livre sur des femmes ?

YM-N : En vivant ma vie, j'ai traversé beaucoup de déserts, des moments pas faciles et, à chaque étape, ma route a rencontré celle d'une femme anonyme qui s'accrochait à la vie en tentant au-delà de sa force de soutenir «plus faible » qu'elle. Beaucoup de personnes m'avaient demandées d'écrire mes mésaventures en 1979 sous Bokassa. Moi, j'avais envie de dire « Merci » à toutes celles dont la route a rencontré la mienne et qui ont bien voulu levé les yeux sur moi. C'est ma façon de dire à Dieu que je ne lui en voulais pas de m'avoir donné ce pays de désespoir comme patrie de naissance.

NOI : Depuis la parution de votre livre, avez-vous réalisé votre objectif ?

YM-N : Non, loin de là ! Encore du chemin à faire et le plus dur est devant moi ! Depuis la parution de mon livre, je n'ai pas arrêté de faire des rencontres et continue d'en faire. Beaucoup de ces rencontres changent et changeront ma vie et mes projets. A moi de savoir les apprécier et les faire fructifier.

NOI : Vous avez deux nationalités, française et centrafricaine et vous vivez à Besançon. Comment combinez-vous votre séjour en France et les actualités centrafricaines ?

YM-N : En France, je vis dans une petite ville, dans laquelle, même si j'y ai mis beaucoup de temps, j'ai fini par me sentir chez moi : sereine et apaisée. C'est la deuxième ville après ma ville natale Sibut où je me sens en sécurité et où je me sens bien. La France m'a accueillie quand je suis partie complètement détruite de Bangui, et Besançon m'a permis de recoller les morceaux. C'est comme avoir deux parents : on les aime tous les deux, même s'ils ont des caractères opposés. Je me sens vraiment française et centrafricaine. Dieu l'a décidé ainsi et j'apprécie.

NOI : Dans la narration du livre, vous avez utilisé la première personne singulier et, lorsque vous présentez d'autres personnages féminins, vous leur prêtez la parole et elles se servent de «je » aussi. Pourquoi avez-vous pris cette focalisation?

YM-N : J'ai écrit ce livre en espérant le mettre à la portée du lecteur centrafricain, quel que soit son niveau d'étude. Je le veux dans les villages, les champs et surtout lu par beaucoup de 
femmes sachant à peine lire. A Besançon, c'est ce qui s'est passé. Des personnes qui l'ont acheté et lu, en ont racheté pour offrir à leurs parents ou leurs enfants ou petits-enfants.

NOI : Votre livre a été publié par votre association. C'est-à-dire que vous avez une maison d'édition n'est-ce pas?

YM-N : Je n'avais pas prévu d'argent pour financer l'édition du livre. Finalement, un ami, membre de l'association, a proposé de le financer, puisque j'avais prévu investir une partie du bénéfice dans les projets de l'association. J'ai donc demandé l'autorisation de pouvoir éditer le livre par l'association et cela s'est fait ainsi.

NOI : Très intéressant ! Cette maison d'édition se trouve à Besançon, votre ville d'adoption. N'y a-t-il pas de maisons d'édition en Centrafrique?

YM-N : J'écris beaucoup de choses régulièrement et je n'avais pas le temps de prospecter pour faire éditer le livre. En Centrafrique, nous n'avons pas de grande maison d'édition et, s'il avait fallu le faire éditer en Centrafrique, il aurait fallu que j'y investisse de l'argent et je n'avais pas prévu cela. Je n'avais pas le projet à ce moment-là de me déplacer en Centrafrique, car je travaillais. J'avais un boulot pour survivre et pas de temps à consacrer à la recherche d'une maison d'édition au pays. En faisant comme je l'ai fait à Besançon, ça n'a pas coûté grand-chose, ni demandé des jours d'absence. J'ai choisi ce qu'il y avait de moins compliqué et de moins couteux en ce moment-là. L'imprimerie avec laquelle je travaille, est la moins chère de ma ville et je peux aller les voir quand je veux, c'est plus pratique.

NOI : Il me semble qu'il y a des difficulté à faire publier des œuvres des femmes. Avez-vous constaté ce type de problème?

YM-N : Je ne me suis pas posé la question jusque-là. En France il y a une maison d'édition qui s'est spécialisée dans les œuvres africaines ; j'ai refusé de l'utiliser, car je ne voulais pas être rangée dans une catégorie d'écrivains par rapport à mes origines. Pour moi, un livre n'a pas de frontières, de sexe ou de pays. J'ai la possibilité d'être libre et de pouvoir faire ce que je veux quand je veux, même si mon choix ne m'a pas permis de vendre beaucoup de livres.

NOI : Parlez-nous des écrivaines centrafricaines et des genres littéraires à la République Centrafricaine.

YM-N : J'avoue que je ne me suis pas trop inquiétée de ce problème jusque-là. Il y a une écrivaine qui est Recteur de la Faculté de lettres à Bangui, Madame Deballet, j'ai juste pu lire 
un de ses ouvrages, et une autre écrit en partenariat avec un Français. Je me souciais plus des femmes qui ont du mal à survivre dans la vie de tous les jours et ne me considère pas encore en cause commune avec les écrivaines. Par contre, une fois que l'association aura avancé dans son projet à Sibut auprès des femmes, j'aimerais encourager et aider les jeunes filles ou garçons à écrire et je verrai à ce moment-là le problème d'édition.

NOI : Qui sont les écrivaines centrafricaines qui ont écrit sur la condition féminine centrafricaine?

YM-N : Je n'en connais pas et la République Centrafricaine est malheureusement dans une phase de survie et non de culture. La population n'a pas trop de moyens à mettre dans les livres, même nos journaux ont du mal à se vendre.

NOI : Et les hommes? Quels sont les thèmes courants dans leurs œuvres ?

YM-N : Nos hommes sont très intellectuels, mais pour dire vrai, jusque-là, écrire et éditer ne faisaient pas trop partie de mes soucis. La vie n'est pas facile en France et, depuis presque douze ans, je me souciais plus de gagner de l'argent afin de faire fonctionner l'association et aider les bénéficiaires et leurs enfants à survivre en Centrafrique. Tous mes écrits étaient en général destinés aux adhérents de notre association. C'est sur l'insistance d'un ami que je m'étais décidée à éditer ce livre. Je n'arrive pas à finir mon deuxième comme je l'aurais souhaité, car je ne dispose pas d'assez de temps pour le faire. Je travaille à plein temps et ne peux m'occuper de l'association que pendant les congés ; cela a occasionné un souci de santé, car je ne me suis pas assez reposée et ceci a affaibli mon cœur.

NOI : Revenons à votre livre ; vous avez mentionné l'existence de la polygamie et l'exploitation par le polygame. Pouvez-vous en parlez plus ?

YM-N : Mon père était monogame. Par contre, j'ai connu des couples polygames et j'ai essayé de les observer. Je pense que j'ai des préjugés par rapport à ce choix de vie, même si j'ai connu des exceptions. Je manque de temps pour approfondir le sujet qui est pour le moment secondaire pour moi.

NOI : Maintenant que vous êtes devenue écrivaine, croyez-vous avoir un rôle à jouer dans la société centrafricaine, la société africaine et la communauté mondiale ? Quel est ce rôle ?

YM-N : Je me considère plus comme une humanitaire responsable d'une association que comme écrivaine. Vous savez, la République Centrafricaine est classée parmi les 
derniers pays au monde : sur le plan mondial 159e sur 163 pays pris en compte. Il y a une grave détérioration des conditions de vie de la population. L'espérance de vie est de quarantecinq ans et le taux d'alphabétisation est au-dessous de 50\%. Voici comment Médecins Sans Frontières décrit la situation sanitaire de la République Centrafricaine : «Une capitale, Bangui, qui concentre l'essentiel des structures de santé du pays. Hors de la capitale, il n'y pas ou très peu d'accès aux soins... Les rares structures éparpillées à travers le pays manquent souvent de médicaments et de personnel médical qualifié... Il y a une centaine de médecins pour tout le pays... ». La majorité du pays est occupé par la rébellion. Ma priorité pour le moment est de me battre pour que le pays change de dirigeants sans violence et que les conditions de vie des femmes puissent s'améliorer. Sauver et soigner le corps avant de passer à l'intellectuel. Par contre, j'aimerais qu'un maximum de femmes en Afrique et dans le reste du monde puissent être au courant du drame qui se vit chez les femmes et enfants en Centrafrique. Il ne faut pas que le combat reste national et méconnu. Nous avons besoin de vous et c'est dans ce but que ma plume pourrait jouer son rôle. Si, grâce à l'écriture, je peux faire connaitre ce pays et son drame, ce serait merveilleux. Mon rôle est d'être la porte-parole de la détresse de tout un peuple très peu connu et pas médiatisé sur le plan international.

NOI : Vous savez, le public en dehors de la République Centrafricaine dépendra des œuvres écrites pour connaitre mieux le pays. Quel sera votre contribution et votre rôle à vos lecteurs?

YM-N : La sortie de mon deuxième livre a pris du retard à cause d'un problème de santé qui a perturbé mon emploi du temps. J'espère qu'il répondra à l'attente de mes lecteurs comme vous.

NOI : Etes-vous heureuse de votre roman ? Est-ce que vous sentez une certaine forme de libération par votre écriture?

YM-N : Ce livre m'a fait revivre mon enfance enfouie profondément au fond de moi. Il fallait que je partage cette partie de ma vie. Cela fait plus de trente ans que je vis en France et écrire ce livre c'est comme écrire un testament pour mes lecteurs et mes deux pays.

NOI : Combien de livres avez-vous à l'heure actuelle?

YM-N : J'ai écrit beaucoup des petites nouvelles, mais mon deuxième livre est toujours en « construction » et j'espère que ça ne va pas durer. 
NOI : Quel message avez-vous pour les femmes africaines?

YM-N : A vous, mes sœurs africaines, je vous demande d'entendre notre voix, de nous soutenir, car nous avons besoin de vous. Tendez-nous la main !!!

NOI : Merci.

\section{Conclusion}

La situation des femmes de Centrafrique ne cesse de se dégrader avec les différents conflits militaro politiques. C'est une situation alarmante. D'après Francis Kopaye, les Centrafricaines «sont enrôlées de force dans les combats, elles servent à la fois « d'esclaves sexuelles » et de «bonnes à tout faire ». Elles sont violées, leurs enfants et leurs conjoints enrôlés sinon exécutés dans le pire des cas. Elles sont les premières victimes du sida ». Le livre de Mété-Nguemeu révèle tant de choses inconnues sur la condition féminine du pays, à savoir la prostitution, la maternité précoce, la monoparentalité, la déperdition scolaire, l'oisiveté, le chômage, le viol etc. Selon Kopaye, «le livre de la compatriote Yvonne Mété est excellent!! C'est un ensemble de témoignages. C'est un roman pas une étude sociologique de la gente féminine » (16). La République Centrafricaine vit dans des crises politiques, sociales et économiques. Je suis en train de traduire le roman en anglais pour faire parvenir ces réalités au monde anglophone.

\section{Références}

Centrafrique Sans Frontières (2004). http://www.centraf-sf.org/cadre_fr.htm (11 octobre 2012.

Iloh, Ngozi O. «La condition féminine centrafricaine dans Femmes de Centrafrique : âmes vaillantes au cour brisé de Mété-Nguemeu ». Communication. $15^{\mathrm{e}}$ conférence de l'Association nigériane des enseignants universitaires de français (ANEUF), Abraka (609 novembre 2012).

Kopaye, Francis. (2008). «Quel avenir pour la femme centrafricaine ? 'Femme, nourrice de l'humanité' ». L'arbre à palabre : Tribune de réflexion, http://www.sozowala.coml'arbreàpalabre

Médecins Sans Frontières, http://www.la-croix.com/Actualites/Monde/Rapport-de-medi (12 mars 2013).

Mété-Nguemeu, Yvonne. Femmes de Centrafrique: âmes vaillantes au cour brisé. Besançon : Centrafrique Sans Frontières, 2008. 